\title{
Calculation of the Energy of Capacitors for a Spot Welding Apparatus by a Numerical Method*
}

\author{
Sergei Zuev ${ }^{[0000-0001-7033-1882]}$, Dmitry Varlamov ${ }^{[0000-0002-7742-5037]}$, Anatoly Fironov ${ }^{[0000-}$ \\ 0003-2683-9958], Alexander Kanareykin ${ }^{\text {[0000-0002-9267-9254] }}$, Artem Timoshenko ${ }^{\text {[0000-0002-5559- }}$ \\ ${ }^{6900]}$, Alexander Shvecov ${ }^{[0000-0002-4840-7542]}$, Vitaly Kuksa ${ }^{[0000-0002-4667-4639]}$ \\ Moscow Polytechnic University, 432700, 38 Bolshaya Semenovskaya, \\ Moscow, Russia \\ sergei_zuev@mail.ru, varlamovdemail.ru, \\ fironoveamfconsulting.ru, knsalex@yandex.ru, \\ prokypt@gmail.com, aleksander55_94@mail.ru, \\ ya. vetal11@yandex.ru
}

\begin{abstract}
This article provides information on the development of an adjustable spot welder implemented on the basis of the capacitor principle without a transformer. Also the scientific work considers the calculation of the energy of the capacitors for a spot welding apparatus using modern methods of numerical simulation and programming. The method of calculation is given, as well as a mathematical solution tool. The calculation results are compared with the experiment. Based on the data obtained, according to the results of calculations, work was carried out on a prototype, the result of which was an adjustable spot welder. It allows you to accurately and quickly weld nickel plates to lithium cells, creating full-fledged batteries from them, which are widely used in modern technology, including promising vehicles. The presence of sensors and a screen allows the operator to control the charging process and the direct operation of the unit, which simplifies the use of the device.
\end{abstract}

Keywords: Capacitor energy, Spot welding apparatus, Numerical method, Welding, Discharge time.

\section{Calculation of the energy of capacitors}

\subsection{Formulation of the problem}

An adjustable spot welding apparatus [1], developed by a team of authors, belongs to the type of semiautomatic devices and is implemented in a capacitor method without using a transformer [2]. The discharge for the welding process gives a block of ten electrolytic capacitors with a voltage of $35 \mathrm{~V}$ and a capacity of $10,000 \mathrm{mF}$. This method allowed to reduce the size and increase the safety of the device. The discharge

Copyright (C) 2020 for this paper by its authors. Use permitted under Creative Commons License Attribution 4.0 International (CC BY 4.0). 


\section{$2 \mathrm{~S}$. Zuev et al.}

of the capacitors in this device, and hence the release of the stored energy, occurs almost instantaneously in the interval of 1-3 ms. This minimizes the heat-affected zone in the welded joint. In addition, the simplicity of dosing energy and upsetting force results in a consistently high weld quality. Main advantages the spot welding apparatus: high performance; minimum heat affected zone due to high energy density and short pulse; connection strength; simplicity of technology that does not require highly qualified personnel; uniformity of the mains load at high welding currents. Let's consider the operation of the welding device in more detail.

Features of the developed adjustable spot welding machine and its differences from analogues:

- "Open" code of the microcontroller [3], which allows the completion and optimization of the device software.

- The cheapness of the development. According to the results of the calculations, an

adjustable spot welder costs an average of $29 \%$ cheaper compared to analogues [4].

- Ergonomics and ease of use [5]. The device is quite easy to disassemble, and bend the

corrugated body of the electrodes in the required direction to perform difficult-to-

access work;

- Increased maintainability due to ease of repair and availability of spare parts and components [6].

When preparing microelectronic products for welding with an apparatus, it is necessary to take into account the aspects of manufacturability. So, taking into account the high degree of deformation during welding of trace elements, the uniformity of which ensures the quality and reliability of the joint, it is necessary to achieve equality and uniformity of the thickness of the welded trace elements. It is desirable to reduce (by about 10-30\%) the deformation of microelements, which is explained by the desire to prevent the formation of defects in the contact and a noticeable decrease in the cross section of the microelements being connected.

A distinctive feature of the developed device is a low rate of mass transfer in the electrode-workpiece contact due to the short pulse duration and welding current.

During the creation of the device, the necessary parameters were calculated, such as the resistance of the welding circuit, the discharge current with the shunt, and the discharge current of normal operation. According to the results of these calculations, the safe modes of operation of the device were determined [7].

An electric discharge for carrying out the welding process gives a block of ten electrolytic capacitors with a voltage of $35 \mathrm{~V}$ and a capacity of $10,000 \mathrm{mF}$. Consider the operation of the device in more detail.

After opening the transistors, the block will discharge onto a nickel plate to which the electrodes are pressed [8]. The duration of the operating mode (discharge) is set by the potentiometer R12 and it is $0.1 ; 0.2 ; 0.5 ; 1.0 ; 2 ; 5$ and 10.0 milliseconds (ms).

Welding current pulses, during which heating and welding of products are carried out, can be single and combined. To improve the quality of welding, the device has the ability to form combined pulses, consisting of two or more parts following each other. 
To determine the optimal operating mode of the welding machine [9], the energy stored in the capacitors was calculated. The main characteristics of the device depend exactly on this value, such as, for example, welding current, max welding power [10].

Energy stored in capacitors:

$$
\mathrm{E}=\mathrm{C} \cdot \mathrm{U} 2 / 2=0,1 \cdot 302 / 2=4,5 \cdot 10-4 \mathrm{~kW} \cdot \mathrm{ms} \text {, }
$$

where $\mathrm{C}$ is the capacitance of the capacitor bank, f; where $\mathrm{U}$ - initial voltage value of transition process, $\mathrm{V}$.

\subsection{The sequence of calculation}

The sequence of calculation can be represented as follows.

The transient process [12] of the discharge of the capacitor will be characterized by the transition of the energy [13] of the electric field of the capacitor into the thermal energy of the resistance of the resistor. In general, the formula will look like:

$$
E=\int_{0}^{\infty} I^{2} \cdot R d t=\frac{U^{2}}{R} \cdot \int_{0}^{\infty} e^{\frac{-2 t}{R C}} d t=\frac{C \cdot U_{C}^{2}}{2},
$$

where I is the discharge current of the capacitor bank, A; R - the resistance of the discharge circuit, Ohm; $\mathrm{t}$ - the discharge time, s; Uc - current value of voltage on the capacitor, decreasing exponentially with time, $\mathrm{V}$.

When the resistance in the discharge circuit is equal to $\mathrm{R}=0.015 \mathrm{Ohms}$, we determine the discharge energy in the general form:

$$
E=\frac{U^{2}}{R} \cdot \int_{0}^{t_{n}} e^{\frac{-2 t}{R C}} d t=\frac{30^{2}}{0,015} \int_{0}^{t_{n}} e^{\frac{-2 t}{0,015 \cdot 0,1}} d t=60000 \cdot \int_{0}^{t_{n}} \sqrt[0,002]{e^{-2 x}},
$$

where $t_{n}$ is the discharge time interval, ms.

Draw up a general table 1 "Optimum values for setting the intervals of the discharge time", calculated in accordance with the formula (3).

Table 1. Optimum values for setting discharge time intervals.

\begin{tabular}{|c|c|}
\hline $\boldsymbol{t n}, \boldsymbol{m s}$ & Discharge energy $(\boldsymbol{E}), \boldsymbol{k W} \cdot \boldsymbol{m s}$ \\
\hline $\mathbf{0 , 1}$ & 5,6 \\
\hline $\mathbf{0 , 2}$ & 10,5 \\
\hline $\mathbf{0 , 5}$ & 21,9 \\
\hline $\boldsymbol{1}$ & 33,14 \\
\hline $\mathbf{2}$ & 41,87 \\
\hline $\mathbf{5}$ & 44,94 \\
\hline $\mathbf{1 0}$ & 44,99 \\
\hline
\end{tabular}

Then, in accordance with formula (4), we get table 2 "The remaining percentage of the energy of the full discharge". 


$$
\varepsilon=\frac{E-E\left(t_{n}\right)}{E} \cdot 100 \%,
$$

where $\mathrm{E}(\mathrm{tn})$ is the discharge energy during tn, ms.

Table 2. The remaining percentage of the energy of the full discharge.

\begin{tabular}{|c|c|}
\hline $\boldsymbol{t} \boldsymbol{n}, \boldsymbol{m s}$ & Remaining energy percentage of the full discharge, $\%$ \\
\hline $\mathbf{0 , 1}$ & 87,5 \\
\hline $\mathbf{0 , 2}$ & 76,7 \\
\hline $\mathbf{0 , 5}$ & 51,3 \\
\hline $\mathbf{1}$ & 26,3 \\
\hline $\mathbf{2}$ & 6,95 \\
\hline $\mathbf{5}$ & 0,13 \\
\hline $\mathbf{1 0}$ & 0,0022 \\
\hline
\end{tabular}

Practical use of the spot welder has shown high quality results. Figure 1 shows that the weld spots are smooth and neat. The quantitative sample during the tests showed the absence of lack of penetration, burnout, deep dents, cracks and splashes when creating a spot welded joint. The lithium-ion batteries were fully functional and fully charged. Thus, the developed apparatus can be used in the manufacture for the assembly of microcircuits, if necessary, for welding miniature hinged elements to them.

For products with semi-conductive and non-conductive film coatings [11], a welding voltage pulse is formed, consisting of several unipolar welding pulses with a given shape change pattern, obtained by the energy of one quarter of the supply voltage period. In this case, it is possible to use the mode of the apparatus, when the first impulse provides an electrical breakdown of the coating, and the subsequent impulses provide welding of products.

A number of welded materials are very sensitive to changes in the uniformity of the electrode pressure [14]. A slight change in the geometry of the working part of the electrode, their wear or misalignment can cause a splash of molten metal. To eliminate the undesirable phenomenon, it is possible to use combined electrodes, consisting of metal electrodes, dielectric bushings, which prevent the deviation and loss of alignment of the electrodes. Such electrodes provide better welding of products.

The calculation results are presented in Figure 1.

\section{Conclusion}

The obtained calculation results, both numerical and graphic, can provide important information on the dynamics of the capacitor charge, which can be used by other scientific groups when conducting research in related fields [15]. The conducted review of scientific papers on the research topic showed significant interest from outside groups on this topic [16]. Also, the obtained data can be used to determine the safe operating modes of this type of devices [17], to develop recommendations on the method of thermal inspection of joints obtained by spot welding. Significant defor- 
mations lead to a thinning of the thickness and, as a consequence, a sharp decrease in the mechanical properties of the joint [18]. An essential prerequisite for obtaining a good quality welded joint using the developed apparatus is a clean surface of the products to be contacted with the same properties [19]. Welding defects can be caused by the presence of areas of local anomalous overheating of the alloy and can be associated with the formation of discontinuities in the process of plastic deformation [20], which requires further research.

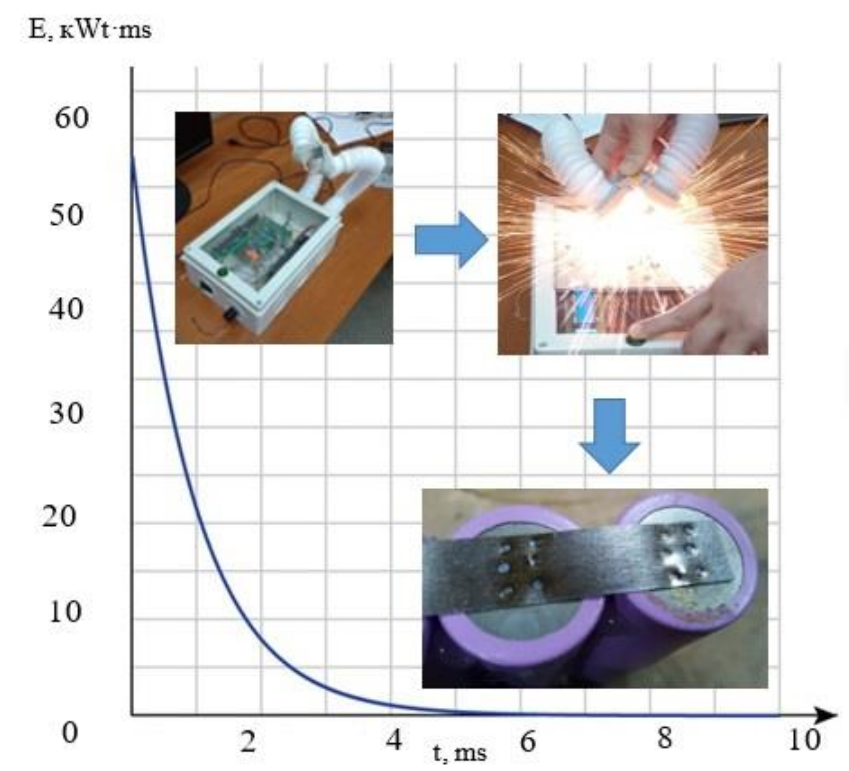

Fig. 1. Discharge energy of the capacitors of the spot welding apparatus. The inset shows the welding process.

\section{References}

1. S.M. Zuev, D.O. Varlamov, V.V. Debelov, A.A. Timoshenko, A.I. Kanareykin, M.A. Peshkun, V.N. Kagdin: Adjustable spot welding machine. Welding and diagnostics. 3, 5862 (2019)

2. Yamamoto N., Liao J., Nakata K.: Friction stir spot welding and resistance spot welding of noncombustible magnesium alloy. Nippon Kinzoku Gakkaishi. 74 (5), 307-313 (2010).

3. Skvortsov, A.A., Rybin, V.V., Zuev, S.M.: Features of electrostimulated degradation of aluminum metallization on silicon surface in the presence of dielectric steps. Technical physics letters. 36(3), 282-284 (2010).

4. Skvortsov, A.A., Orlov, A.M., Zuev, S.M. Diagnostics of degradation processes in the metal-semiconductor system. Russian Microelectronics. 41(1), 31-40 (2011).

5. Han L., Thornton M., Boomer D., Shergold M.: Effect of aluminum sheet surface conditions on feasibility and quality of resistance spot welding. Journal of Materials Processing Technology. 210 (8), 1076-1082 (2010). 
$6 \mathrm{~S}$. Zuev et al.

6. Skvortsov, A.A., Zuev, S.M., Koryachko, M.V.: Non-stationary phase transitions in systems metallization of silicon structures. Russian Microelectronics. 45 (3), 215-222 (2016).

7. Akbolatov E.Z., Kiselev A.S., Slobodyan M.S.: Prediction and stabilization of initial resistance between electrodes for small-scale resistance spot welding. Welding in the World. 63 (2), 443-457(2019).

8. Skvortsov, A.A., Zuev, S.M., Koryachko, M.V.: Contact melting of aluminum-silicon structures under conditions of thermal schock. Key Engineering Materials. 771, 118-123 (2018).

9. Skvortsov, A.A., Zuev, S.M., Koryachko, M.V., Voloshinov, E.B.: Deflected state of semiconductor near-contact region at electrodegradation of metallization track on its surface. Periodico Tche Quimica. 16 (33), 707-715 (2019).

10. Skvortsov, A.A., Koryachko, M.V., Zuev, S.M., Demchenkova, A.A.: Melt drops movement over semiconductor. Periodico Tche Quimica. 16 (33), 681-687 (2019).

11. Skvortsov, A.A., Koryachko, M.V., Zuev, S.M., Skvortsova, A.A.: The effect of thin dielectric layers at silicon on interconnection heating dynamics at thermal shocks. Periodico Tche Quimica. 16 (3)3, 448-456 (2019).

12. Zhang H.-F., Wang X., Qian Q., Li J.-W., Liu H.-X.: Technological experiments of laser high-speed impact spot welding. Guangxue Jingmi Gongcheng. 23 (2), 297-304 (2015).

13. Zuev, S.M., Fironov, A.M., Varlamov, D.O., Kuksa V.V.: The search of new diagnosing and predicting methods to the thermomechanical and strength characteristics of the component base of control systems for unmanned vehicles of the "smart city" based on 5G technologies. Journal of Physics: Conference Series, 1347, 783-787 (2019).

14. Aslanlar S., Ogur A., Ozsarac U., Ilhan E.: Welding time effect on mechanical properties of automotive sheets in electrical resistance spot welding. Materials and Design. 29 (7), 1427-1431 (2008).

15. Turgutlu A., Al-Hassani S.T.S., Akyurt M.: Experimental investigation of deformation and jetting during impact spot welding. International Journal of Impact Engineering. 16 (5-6), 789-799 (1995).

16. Fayzimatov U., Sheng B., Xiao Z., Toure I.: Reliability analysis and maintenance optimization of projection spot welding machine in the automotive industry. International Journal of Performability Engineering. 14 (8), 1833-1841 (2018).

17. Kapil A., Lee T., Vivek A., Daehn G., Cooper R., Hetrick E.: Spot impact welding of an age-hardening aluminum alloy: process, structure and properties. Journal of Manufacturing Processes. 37, 42-52 (2019).

18. Zhang H.-F., Wang X., Qian Q., Li J.-W., Liu H.-X.: Technological experiments of laser high-speed impact spot welding. Guangxue Jingmi Gongcheng. 23 (2), 297-304 (2015).

19. Skidanenko A.V.: Modeling heat-transfer processes of resistance spot welding. Bulletin of the Russian Academy of Sciences: Physics. 80 (6), 742-745 (2016).

20. Sadowski T., Golewski P., Kneć M.: Experimental investigation and numerical modelling of spot welding-adhesive joints response. Composite Structures. 112 (1). 66-77 (2014). 